Rev Med (São Paulo). 2014 abr.-jun.;93(2):56-62.

DOI: http://dx.doi.org/10.11606/issn.1679-9836.v93i2p56-62

\title{
Infecções e emoções
}

\author{
Infections and emotions
}

Marcos Boulos

Boulos M. Infecções e emoções. Rev Med (São Paulo). 2014 abr.-jun.;93(2):56-62.

RESUMO: O presente artigo mostra através de revisão da literatura pertinente sobre a interação mente-corpo, de como as alterações emocionais como estresse, angústia, depressão, levam a alteração da imunidade com consequente predisposição ao surgimento de doença física.

DESCRITORES: Infecção/complicações; Infecção/psicologia; Emoções manifestas/fisiologia; Psicofisiologia; Terapias mentecorpo/psicologia.
ABSTRACT: This paper based on review of the literature about mind body interactions, show us the role of emotional alterations like stress and depression in diminishing the immunity and causing a body disease.

KEYWORDS: Infections/complications; Infections/psychology; Expressed emotion/physiology; Psychophysiology; Mind-body therapies/psychology.

Professor Titular de Moléstias Infecciosas e Parasitárias da Faculdade de Medicina da Universidade de São Paulo.

Endereço para correspondência: Av. Dr. Arnaldo, 455 - Cerqueira César. CEP: 01246903 - São Paulo, SP, Brasil. E-mail: mboulos@usp.br 


\section{INTRODUÇÃO}

$\mathbf{O}$ modelo científico aplicado à Medicina teve enormes vantagens para o reconhecimento das causas e dos mecanismos causadores de doenças além de ter possibilitado diagnósticos rápidos e eficientes e auxiliar no tratamento através da confecção de medicamentos cada vez mais eficazes.

Por outro lado, a concentração de esforços para desvendar diferentes aspectos biomédicos fez com que, gradativamente nos afastássemos da relação humana propriamente dita e, com frequência, o ato médico passou a ter um objetivo, ou seja, tratamos o paciente como objeto a ser reparado.

Esta visão mecanicista do indivíduo trouxe vieses irreparáveis na formação do profissional de saúde e na relação médico-paciente.

Hoje inclusive, a maior parte dos profissionais de saúde se sente desconfortável quando não é necessário realizar intervenções "científicas", ou seja, quando o "paciente" vem apenas solicitar uma informação, pedir um conselho, ou apenas para ser ouvido pelo doutor.

Várias questões importantes para uma abordagem adequada ao indivíduo doente e que não encontram suporte no modelo científico tem instigado o médico observador a repensar parte do conhecimento aprendido durante sua formação.

Porque o otimista evolui melhor que o pessimista?

Observações clínicas mostram a todo médico atento, distintas evoluções de doenças similares independente da conduta instituída. O reconhecimento de que o indivíduo que acredita, o otimista evolui melhor que aquele que não acredita ou o pessimista está na base do entendimento humano e não só para questões de saúde.

A busca de dados que possam corroborar essa milenar observação, principalmente levando em consideração o modelo biomédico que prevalece no pensar científico, que a princípio era impensável por levar em consideração dados qualitativos, hoje se torna mais disponível pelo maior volume de informações a respeito da interação corpo mente.

O estresse pode causar infecção?

Hoje, inúmeros dados demonstram essa assertiva e não só quanto a doenças infecciosas, mas também para outras doenças como câncer, doenças cardiovasculares, hipertensão entre outras.

Na história da Medicina, essas e outras questões eram tratadas de forma natural, pois o ser humano era visto como uma unidade inseparável, como dizia Platão:

\begin{abstract}
"O tratamento do corpo pelo médico não é possivel sem o simultâneo tratamento da alma; mais ainda, que talvez nem sequer isto baste: que, talvez, até seja impossivel fazê-lo sem o conhecimento do ser em seu todo".
\end{abstract}

Esta visão foi gradativamente sendo sacrificada em busca de explicação para todos os eventos da natureza principalmente com a introdução do modelo científico há alguns séculos atrás, e teve seu ápice com Descartes e seu famoso dualismo:

\begin{abstract}
"Meu corpo, como posso ver claramente, é uma substância. É uma substância material, assim como minha alma é uma substância pensante. A coisa chamada eu, consiste de duas partes distintas - a máquina que se move, ou o corpo, e a mecânica que pensa, ou a alma" 2 .
\end{abstract}

Esta visão dualista teve seu ápice no fim do século XIX com a assunção do materialismo como a única possibilidade a ser acreditada:

"No fim do século XIX, cientistas sociais e do
comportamento, adotaram a teoria do conhecimento
e o método das ciências fisicas. A maioria dos
cientistas então defendia a noção exclusivamente
material da ciência, sendo incapazes de distinguir
ciência de cientificismo que advogava nada existir
fora da matéria. Com a adoção do cientificismo,
tudo o que era exclusivamente humano como amor,
compaixão, desejo, espiritualidade, não só foram
intencionalmente omitidos dos questionamentos, eles
foram inteiramente eliminados da vida" .

Com a evolução da Física e as limitações impostas à Mecânica Newtoniana por Einstein e a teoria quântica de Bohr e Heisenberg a visão materialista da ciência sofreu um abalo, principalmente após a Teoria da Incerteza de Heisenberg que mostrava que o observador participa do resultado final de toda experiência mostrando que o sujeito interfere em todos os eventos.

A partir desses dados ficou evidenciado o milenar conhecimento da unidade entre sujeito e objeto e da importância da crença para que os resultados sejam obtidos.

Daí vem a necessidade das pesquisas humanas serem duplo cegas (nem o médico nem o paciente devem saber qual medicamento está sendo administrado, pois os dois interferem no resultado final), e ação do placebo. otimista.

Fica aqui então evidenciada a melhor evolução do

Para uma análise mais pormenorizada de alguns trabalhos científicos que mostram essa interação faremos uma pequena revisão da literatura.

Inicialmente abordaremos as doenças infecciosas para, a seguir, apresentarmos dados de outras doenças e também de alterações devido ao meio ambiente e às relações sociais.

\section{CASUÍSTICA}

Este trabalho é baseado na revisão da literatura pertinente.

\section{Infecções}


Brown em $1993^{4}$ realizou um estudo onde injetou colônias de Mycobacterium avium em peritônio de camundongos e dividiu-os em quatro grupos distintos: o primeiro grupo foi controle, nos segundo, terceiro e quarto grupos submeteu os camundongos a situação de estresse, ou seja, imobilizou-os e girava-os em uma, cinco ou dez vezes (grupos 2, 3 e 4 sucessivamente) sacrificando-os a seguir. A quantidade de unidades formadoras de colônias foi maior no grupo 4 e a seguir no 3 tanto no pulmão como no baço dos camundongos. Não houve diferenças significativas entre o grupo controle e o 2 mostrando que um "pequeno" estresse não interferiu na imunidade dos camundongos avaliados (Figuras 1 e 2).
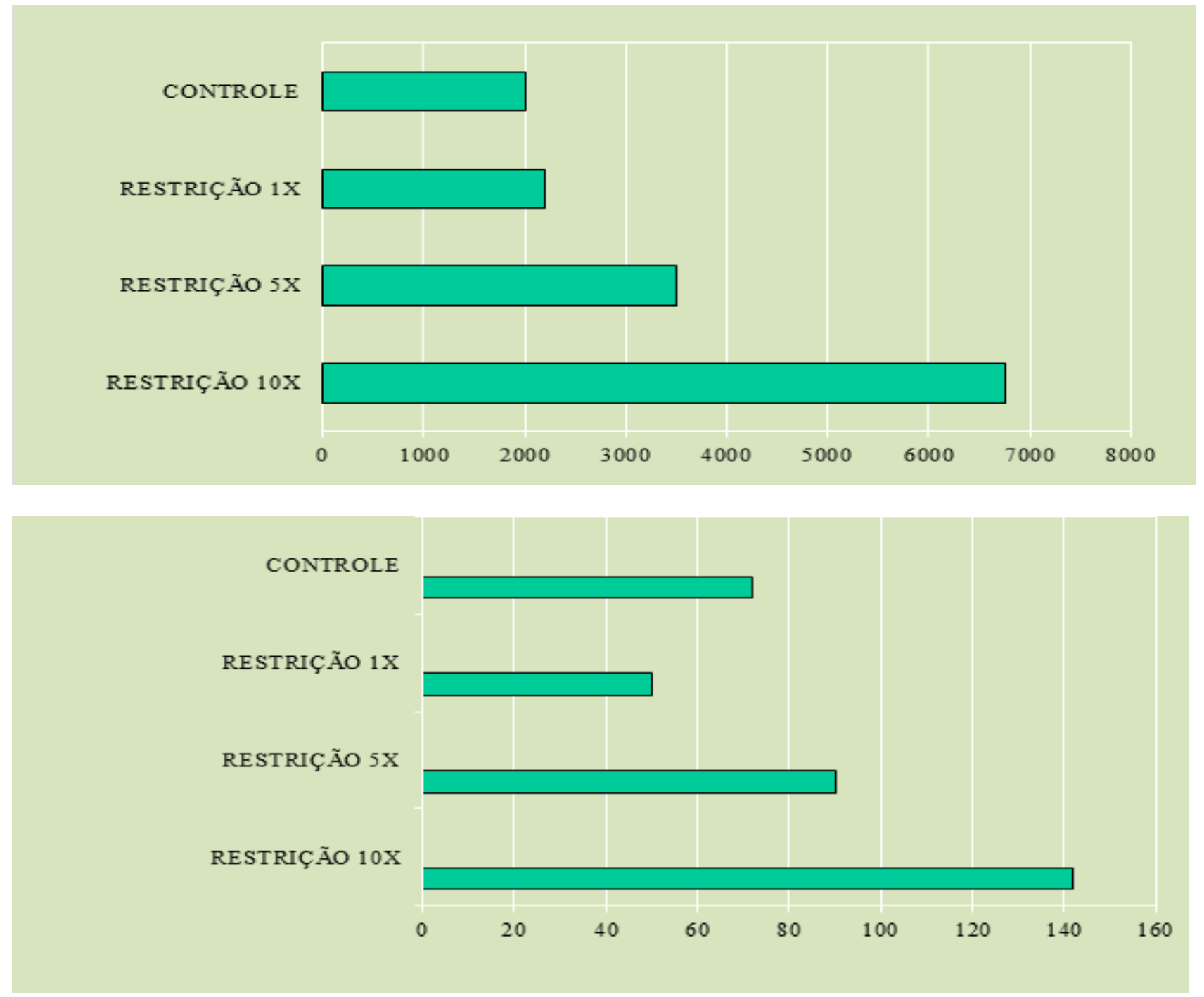

Figuras 1 e 2. Estresse, depressão estimulam o hipotálamo que através de uma cadeia de reações estimula a pituitária e a seguir a adrenal que produz cortisol que juntamente com a adrenalina e noradrenalina inibem a produção de fatores responsáveis pela defesa do indivíduo levando a consequente diminuição da imunidade e doença.

Reativação da infecção pelo vírus Epstein Barr (EBV) foi observada em cadetes americanos e estudantes quando submetido a estresse $\mathrm{e}^{5,6}$.

Em trabalho prospectivo Cohen, após avaliar o grau de estresse na população analisada, administrou gotas nasais contendo 1 de 5 vírus respiratórios e observou que as taxas de infecção respiratória $(\mathrm{p}<0,005)$ e resfriado clínico $(\mathrm{p}<0,02)$ aumentaram com aumento do estresse e que a associação observada foi similar para os 5 tipos de vírus ${ }^{7}$.
É na infecção pelo HIV que muitos estudos se concentraram com destaque para estudo prospectivo de Leserman et al. ${ }^{8}$ que acompanharam pacientes infectados durante 9 anos com várias publicações no período sendo que após esse período com 96 indivíduos acompanhados concluíram que maior média cumulativa de eventos estressantes e menor média cumulativa de suporte social foram preditores de mais rápida progressão para AIDS. Ickovics et al. ${ }^{9}$ acompanharam 765 mulheres entre 16 
e 55 anos de idade em estudo de coorte, longitudinal, prospectivo de 1993 a 1995 com seguimento até março de 2000 e concluíram que mulheres com sintomas depressivos crônicos tiveram 2 vezes mais chance de morrer (RR 2,$0 ; 95 \%$ IC) e também estiveram associados com maior diminuição de linfócitos CD4.

\section{Outras doenças}

A literatura médica tem abordado com cada vez maior frequência as interações entre fatores emocionais e o surgimento de câncer, melhora da doença já diagnosticada e mortalidade.

Em uma avaliação longitudinal da associação entre estresse e câncer usando métodos meta-analíticos Chida et al. ${ }^{10}$ mostraram que fatores psicossociais que induzem ao estresse foram relacionados à incidência de câncer em população previamente saudável em 165 estudos ( $\mathrm{p}<$ 0,005), a pior prognóstico em câncer já diagnosticado em 330 estudos $(\mathrm{p}<0,001)$ e à maior mortalidade por câncer em 53 estudos $(\mathrm{p}<0,001)$.

Em um estudo caso-controle de observação da relação entre fatores psicossociais e câncer de pulmão realizado na China com 750.000 participantes observouse que a incidência de câncer de pulmão esteve associada com três fatores: explosões emocionais não controladas, circunstâncias inadequadas de trabalho incluindo relação ruim com companheiros e depressão por longo período de tempo ${ }^{11}$.

Trabalho observacional realizado entre pessoas que imigraram para Israel antes e após a II Guerra Mundial mostrou incidência maior de todos os cânceres, principalmente de mama e colo retal, naquelas que participaram da guerra ${ }^{12}$.

Em estudo caso-controle em 50 mulheres que tiveram sua primeira recorrência após cirurgia de câncer de mama e 50 mulheres em remissão após a cirurgia observouse que maior dificuldade de lidar com graves eventos de vida esteve significativamente associada com a primeira recorrência do câncer de mama - RR 5,67 (1,57-37,20) ${ }^{13}$.

Desequilíbrio emocional não está relacionado unicamente a surgimento de doenças, mas também a uma vida sem motivação levando, inclusive, a morte mais precoce como pode ser atestado pelos três trabalhos seguintes.

Young ${ }^{14}$ observou mortalidade maior em viúvos após 6 meses da morte da esposa.

Em acompanhamento da comunidade de Alameda County, por 9 anos nos Estados Unidos, Berkman e Syme ${ }^{15}$ observaram mortalidade 2,3 vezes maior para homens e 2,8 vezes maior para mulheres com menor suporte social.

Uma revisão meta-analítica entre relação social e risco de mortalidade em 148 estudos com 308.849 participantes mostrou $50 \%$ de aumento da probabilidade de sobrevida para aqueles com relações sociais intensas
$(\mathrm{OR}=1,50)^{16}$ tendo como conclusão que a influência das relações sociais para o risco de mortalidade é comparável com fatores de risco bem estabelecidos para a mesma.

Vários trabalhos mostram também a melhora da doença já estabelecida com intervenções para diminuição do estresse.

Stone ${ }^{17}$ mostrou que 6 meses de relaxamento levaram a diminuição da hipertensão arterial com diminuição da atividade adrenérgica periférica em pacientes por ele acompanhados.

Segal et al. ${ }^{18}$ conduziram estudo na cidade de Toronto onde comparam o emprego de meditação e farmacoterapia na recidiva de quadros depressivos. Pacientes que apresentaram remissão após 8 meses de tratamento antidepressivo foram randomizados para receberam ou medicação de manutenção, ou participarem de grupo de terapia cognitiva receberam ou placebo, sendo seguidos por 18 meses. Pacientes do grupo de terapia cognitiva e dos que receberam tratamento de manutenção apresentaram $73 \%$ menor risco de recidiva $(p=.03)$ comparados com placebo.

Trabalho semelhante foi realizado em abordagem a pacientes com esclerose múltipla com resultados também alentadores ${ }^{19}$.

Estudo de neuroimagem utilizando a ressonância magnética em 16 indivíduos saudáveis que participaram de grupo com práticas de meditação por 8 semanas mostrou aumento da concentração de substância cinzenta no hipocampo esquerdo. Houve aumento também no cingulado posterior do córtex, na junção têmporo-parietal e no cerebelo dos meditadores quando comparados com controles $^{20}$.

\section{DISCUSSÃO}

Na área da saúde, o reconhecimento da interação corpo-mente com repercussões no surgimento e recuperação de doenças já data de algumas décadas e essa interação pode ser mostrada pelo esquema abaixo onde podemos visualizar a conexão entre emoções positivas e aumento da imunidade e emoções negativas e diminuição da imunidade.

Um exemplo da importância do otimismo na evolução de doenças, o efeito placebo, está bem mostrada no trabalho de Moseley et al. ${ }^{21}$ em 180 pacientes com osteoartrite de joelho randomizados em 3 grupos: debridamento artroscópico, lavagem por artroscopia e cirurgia placebo, acompanhados por 24 meses através 5 escalas auto referidas ( 3 para dor e 2 funcionais); todos os grupos obtiveram melhora, não havendo diferença estatística entre eles!

A ênfase de como a mente pode interferir no corpo é bem evidenciada nos trabalhos onde são realizadas preces à distância para beneficiar pessoas doentes, ou seja, como podemos interferir, com nossa vontade, na vida de outra pessoa. Dois estudos que utilizaram a prece intercessória 
em pacientes hospitalizados em unidades coronarianas foram realizados nos Estados Unidos ${ }^{22,23}$ e mostraram melhoras de vários parâmetros na saúde dos indivíduos que recebiam a prece (e não sabiam que recebiam) comparados com igual número dos que não a receberam.

O mesmo tipo de estudo foi realizado em pacientes com AIDS na cidade de São Francisco, Estados Unidos, e também revelou resultados benéficos aos pacientes alvo de preces a distância ${ }^{24}$.

Outro estudo surpreendente com a utilização de preces à distância foi feito na Coréia para avaliar o efeito da prece na taxa de gestação em mulheres fertilizadas "in vitro". Em tal estudo as pessoas que realizavam as preces estavam em outros continentes e ninguém no hospital onde foi realizado o estudo sabia do mesmo (com exceção do autor do estudo). $\mathrm{O}$ grupo que recebeu as preces teve maior taxa de gestação $(50 \times 26 \%)$ e maior taxa de implantação $(16,3 \times 8 \%)^{25}$.

Todos os trabalhos referidos respeitaram toda metodologia científica e evidenciaram a importância da relação mente corpo para uma vida mais saudável e mais, da importância da relação humana para vivermos bem, com menos doenças e mais felizes.

A interação observada entre seres humanos não é exclusiva. Esta interação também é observada com animais $^{26}$ ou plantas ${ }^{27}$.

O adequado equilíbrio da interação corpo-mente é a chave para o bem estar bio-psico-social, de um repensar sobre nossa vida e de nossa relação conosco e com o outro, como dizia Albert Einstein:

\begin{abstract}
"O ser humano é parte de um todo, chamado por nós "universo", uma parte limitada no tempo e espaço. Ele sente seus pensamentos e sentimentos como algo separado do resto - uma espécie de ilusão de ótica de sua consciência. Esta ilusão é tal qual uma prisão para nós, restringindo-nos às nossas decisões pessoais e afeto a poucas pessoas ao nosso redor".
\end{abstract} reflexões.

Queria dar um testemunho e a seguir, fazer algumas

Certa vez, cuidando de um paciente com AIDS em situação clínica bastante difícil com mais de uma infecção oportunista e com imunidade bastante diminuída $\left(\mathrm{CD}_{4}\right.$ $=4$ ), ouvi dele que ficaria bom apesar das dificuldades momentâneas. Ele de fato melhorou e depois de alguns meses a imunidade começou a subir e as infecções oportunistas foram controladas. É importante destacar que naquele momento não existiam medicamentos com eficácia comprovada para a infecção pelo HIV.

Passado algum tempo, numa consulta de retorno, ele me fez uma declaração surpreendente: "doutor, eu dou graças a Deus por ter AIDS". Percebendo minha perplexidade ele continuou: "quando recebi o diagnóstico que estava infectado pelo HIV vivia de expedientes, na rua, drogado, sem amigos ou perspectivas, agredia as pessoas por nada, inclusive meus pais e acreditava estar bem comigo mesmo. De repente percebi que iria morrer! Questionei minha vida, aonde queria chegar, e mais, quem era eu? E meus sonhos? Comecei a beijar as pessoas, pois não sabia se iria as ver mais, não mais reagi ao que considerava provocações, que importância teria o dito na eminência da morte? Olhava para as pessoas para reconhecê-las, talvez pela última vez. Comecei a ter amigos, consegui um bom trabalho, as pessoas confiam em mim, estou feliz, percebi como a vida pode ser maravilhosa. Como amaldiçoar a causa de meu bem estar?"

Quase três décadas depois este paciente continua me visitando no consultório. Sua imunidade é próxima da normalidade; teve um longo caminho produtivo de realizações, alegrias e dissabores nesse período e vários amigos e parentes morreram no período.

Por que precisamos ver "a morte de perto" para podermos viver integralmente? Por que adiamos continuamente a realização de nossos sonhos?

Sim, somos mortais, e precisamos viver como tal.

Precisamos parar de viver o passado que não existe e ficar imaginando um futuro que não existe. Quem garante que acordaremos amanhã? A única realidade é o presente. Precisamos vive-lo com concentração, com amor, com dedicação para que nossos atos tenham a importância que deles esperamos e viver a vida integralmente.

Se não estivermos atentos e dedicados para nossas relações, e nos colocarmos no lugar do outro quando necessário, dificilmente viveremos uma vida plena com amor.

Os profissionais de saúde devem atentar para o fato que o objeto de sua ação também é sujeito, e que só podemos conduzir nossa ação profissional competentemente se ouvirmos com atenção as dores de quem nos procura. Afinal, quem sabe mais da doença o médico ou o paciente? Se o médico a conhece por estuda-la o paciente a está sentindo. É dos saberes complementares que se constrói a cura.

As ciências que cuidam nunca deveriam ser chamadas de biológicas, pois o conhecimento da biologia não faculta ninguém a cuidar profissionalmente de outra pessoa. São ciências humanas com base na biologia. A biologia nos dá a base do conhecimento para que possamos tratar competentemente o paciente que nos procura, mas só na interação entre sujeitos é que o processo de transformação ocorre.

Não é incomum a ocorrência de alunos bastante qualificados durante a graduação não conseguirem ser médicos clínicos "badalados" por falta de uma relação humana mais consistente. $\mathrm{O}$ oposto também ocorre: estudantes medianos que se transformam em médicos clínicos reconhecidos por sua boa relação humana.

Gadamer em seu livro: O estado oculto da saúde descreve em outras palavras:

"O poder da medicina moderna é deslumbrante. Não obstante, apesar de todos os progressos sobre 
enfermidade e saúde que nos tem chegado das ciências naturais, e apesar da enorme inversão efetuada em técnicas racionalizadas que permitem o diagnóstico e tratamento, o terreno do não racional segue sendo particularmente amplo. Isto se volta evidentemente, por exemplo, ao fato de que o conceito de médico eficaz e até genial se aproxima - como em todos os tempos - mais a de um artista do que a um homem da ciência. Por isto, neste aspecto mais do que qualquer outro, é impossivel negar o indispensável e o digno que é buscar a experiência prática" ${ }^{28}$.

A habilidade de estar junto, de ouvir com atenção, de olhar nos olhos, de poder sentir em si as dores do

\section{REFERÊNCIAS}

1. Platão. Fedro. In: Platão diálogos Socráticos III. Bauru: Edipro; 2008.

2. Descartes R. Meditações metafísicas. São Paulo: Martins Fontes; 2005.

3. Fahlberg LL, Fahlberg LA. Exploring spirituality and consciousness with an expanded science: beyond the ego with empiricism, phenomenology, and contemplation. Am J Health Promot. 1991;5(4):273-81. doi: 10.4278/0890-1171-5.4.273.

4. Brown DH, Sheridan J, Pearl D, Zwilling BS. Regulation of mycobacterial growth by the hypothalamus-pituitaryadrenal axis: differential responses of Mycobacterium bovis BCG-resistant and -susceptible mice. Infect Immun. 1993;61(11):4793-800. Available from: http://iai.asm.org/ content/61/11/4793.long.

5. Glaser R, Friedman SB, Smyth J, Ader R, Bijur P, Brunell P, Cohen N, Krilov LR, Lifrak ST, Stone A, Toffler P. The differential impact of training stress and final examination stress on herpesvirus latency at the United States Military Academy at West Point. Brain Behav Immun. 1999;13(3):24051. doi: 10.1006/brbi.1999.0566.

6. Yang EV, Glaser R. Stress-induced immunomodulation: impact on immune defenses against infectious disease. Biomed Pharmacother. 2000;54(5):245-50. doi: 10.1016/ S0753-3322(00)80066-9.

7. Cohen S, Tyrrell DA, Smith AP. Psychological stress and susceptibility to the common cold. N Engl J Med. 1991;325(9):606-12. doi: 10.1056/NEJM199108293250903.

8. Leserman J1, Petitto JM, Gu H, Gaynes BN, Barroso J, Golden RN, Perkins DO, Folds JD, Evans DL. Progression to AIDS, a clinical AIDS condition and mortality: psychosocial and physiological predictors; Psychol Med. 2002;32(6):1059-73. doi: http://dx.doi.org/10.1017/S0033291702005949.

9. Ickovics JR, Hamburger ME, Vlahov D, Schoenbaum outro dá ao médico a possibilidade de interagir na busca da recuperação do paciente, e mais, o transforma em um indivíduo fundamental numa sociedade em transformação.

\section{CONCLUSÕES}

A saúde está relacionada ao bem estar bio-psicosocial e consequentemente a doença está relacionada a alterações desse trinômio. A compreensão que, para cuidarmos de um indivíduo, precisamos estar atentos para seu corpo, mente e ambiente que vive é fundamental para que possamos ser competentes em nossa ação e possamos ajudá-lo a redirecionar sua vida.

EE, Schuman P, Boland RJ, Moore J, HIV Epidemiology Research Study Group. Mortality, CD4 cell count decline, and depressive symptoms among HIV-seropositive women: longitudinal analysis from the HIV Epidemiology Research Study. JAMA. 2001;285(11):1466-74. doi:10.1001/ jama.285.11.1466.

10. Chida Y1, Hamer M, Wardle J, Steptoe A. Do stress-related psychosocial factors contribute to cancer incidence and survival? Nat Clin Pract Oncol. 2008;5(8):466-75. doi: $10.1038 /$ ncponc 1134 .

11. Fan RL, Zheng SH, Wu ZS. Study on the relationship between lung cancer at preclinic stage and psycho-social factor. A case-control study. Zhonghua Liu Xing Bing Xue Za Zhi. 1997;18(5):289-92.

12. Keinan-Boker L, Vin-Raviv N, Liphshitz I, Linn S, Barchana M. Cancer incidence in Israeli Jewish survivors of World War II. J Natl Cancer Inst. 2009;101(21):1489-500 . doi: 10.1093/ jnci/djp327.

13. Ramirez AJ, Craig TK, Watson JP, Fentiman IS, North WR, Rubens RD. Stress and relapse of breast cancer. BMJ. 1989;298(6669):291-3. doi: http://dx.doi.org/10.1136/ bmj.298.6669.291.

14. Young M, Benjamin B, Wallis C. Mortality of widowers. Lancet. 1963;282:454-6. doi: 10.1016/S0140-6736(63)92193-7

15. Berkman LF, Syme SL. Social networks, host resistance, and mortality: a nine-year follow-up study of Alameda County residents. Am J Epidemiol. 1979;109(2):186-204.

16. Holt-Lunstad J, Smith TB, Layton JB. Social relationships and mortality risk: a meta-analytic review. PLoS Med. 2010;7(7):e1000316. doi:10.1371/journal.pmed.1000316.

17. Stone RA, De Leo J. Psychotherapeutic control of hypertension. N Engl J Med. 1976;294:80-4. doi: 10.1056/ NEJM197601082940204. 
18. Segal ZV, Bieling P, Young T, MacQueen G, Cooke R, Martin L, Bloch R, Levitan RD. Antidepressant monotherapy vs sequential pharmacotherapy and mindfulness-based cognitive therapy, or placebo, for relapse prophylaxis in recurrent depression.; Arch Gen Psychiatry. 2010;67(12):1256-64. doi: 10.1001/archgenpsychiatry.2010.168.

19. Grossman P, Kappos L, Gensicke H, D'Souza M, Mohr DC, Penner IK, Steiner C. MS quality of life, depression, and fatigue improve after mindfulness training: a randomized trial. Neurology. 2010;75(13):1141-9. doi: 10.1212/ WNL.0b013e3181f4d80d.

20. Hölzel BK, Carmody J, Vangel M, Congleton C, Yerramsetti SM, Gard T, Lazar SW. Mindfulness practice leads to increases in regional brain gray matter density. Psychiatry Res Neuroimaging. 2011;191(1):36-43. doi: 10.1016/j. pscychresns.2010.08.006

21. Moseley JB, O’Malley K, Petersen NJ, Menke TJ, Brody BA, Kuykendall DH, Hollingsworth JC, Ashton CM, Wray NP. A controlled trial of arthroscopic surgery for osteoarthritis of the knee; N Engl J Med. 2002;347(2):81-8. doi: 10.1056/ NEJMoa013259.

22. Byrd RC. Positive therapeutic effects of intercessory prayer in a coronary care unit population. South Med J. 1988;81(7):826-9.
23. Harris WS, Gowda M, Kolb JW, Strychacz CP, Vacek JL, Jones PG, Forker A, O'Keefe JH, McCallister BD. A randomized, controlled trial of the effects of remote, intercessory prayer on outcomes in patients admitted to the coronary care unit.; Arch Intern Med. 1999;159(19):2273-8. doi:10.1001/archinte.159.19.2273

24. Sicher F, Targ E, Moore D 2nd, Smith HS. A randomized double-blind study of the effect of distant healing in a population with advanced AIDS. Report of a small scale study. West J Med. 1998;169(6):356-63. Available from: http://www.ncbi.nlm.nih.gov/pmc/articles/PMC1305403/ pdf/westjmed00327-0028.pdf

25. Cha KY, Wirth DP. Does prayer influence the success of in vitro fertilization-embryo transfer? Report of a masked, randomized trial. J Reprod Med. 2001;46(9):781-7.

26. Nerem RM, Levesque MJ, Cornhill JF. Social environment as a factor in diet-induced atherosclerosis; Science. 1980;208(4451):1475-6. doi: 10.1126/science.7384790.

27. Backster C. O mundo secreto das plantas. 1966.

28. Gadamer H-G. El estado oculto de la salud. Barcelona: Editorial Gedisa; 2001. 